\title{
Conditions for detectability in distributed consensus-based observer networks
}

\author{
V. Ugrinovskii
}

\begin{abstract}
The paper discusses fundamental detectability properties associated with the problem of distributed state estimation using networked observers. The main result of the paper establishes connections between detectability of the plant through measurements, observability of the node filters through interconnections, and algebraic properties of the underlying communication graph, to ensure the interconnected filtering error dynamics are stabilizable via output injection.
\end{abstract}

\section{INTRODUCTION}

An emerging trend in the area of distributed estimation is concerned with the development of consensus-based distributed filtering algorithms to allow each node to carry out estimation by reaching a consensus with its neighbours. An interest in this topic is due to advantages that distributed processing of measurements in sensor networks offers, over transmitting the raw data.

A number of sufficient conditions have been proposed recently to address the design of such algorithms [9], [10], [12]. These conditions typically make use of Linear Matrix Inequalities or matrix Riccati equations and inequalities to guarantee a suboptimal level of filter performance and/or consensus performance between node estimators. However, the problem of establishing feasibility of these LMI/Riccati design conditions from graph theoretical and systems theoretical viewpoints remains an essentially open problem.

In this paper, we consider the detectability problem for a distributed state estimator which observes a linear plant through a network of interconnected filters. The problem is related to a large class of distributed estimation problems that employ interconnected observers, such as Kalman filters or $H_{\infty}$ filters. In particular, we are interested in the situation where the plant is not detectable from individual node's measurements. For example, multi-vehicle Simultaneous Localization and Mapping (SLAM) problems exhibit this feature; see the example in Section [I-B] and [4]. It was alluded in [10] that in such situations the nodes must rely on interconnections to ensure the state estimation problem is feasible. This paper presents a rigorous analysis of this claim.

Our chief objective is to establish conditions which guarantee detectability of a large scale system describing observer error dynamics in consensus based distributed estimation problems. Such a detectability property is necessary for these

This research was supported under Australian Research Council's Discovery Projects funding scheme (Project number DP120102152).

School of Engineering and IT, University of NSW at the Australian Defence Force Academy, Canberra, Australia, Email: v.ugrinovskii@gmail.com. Part of this work was carried out during the author's visit to the Australian National University. estimation problems to have a solution. The main results in this paper characterize the detectability property of this large scale system in terms of detectability properties of its components. Namely, we present a necessary condition for the large scale detectability expressed in terms of the 'local' detectability of the plant through individual filter measurements, and the observability properties of the node filters through interconnections. Secondly, we show that these local properties are also sufficient for the distributed detectability property to hold if the communication graph has a spanning tree. We also extend these results to a more general case where the graph is weakly connected but is not spanned by a tree, and show that in this case the problem reduces to establishing distributed detectability of certain clusters within the system. For this, we also give necessary and sufficient conditions.

Our results show that in the distributed estimation scenario, the algebraic properties of the graph Laplacian must be complemented by observability properties of the node filters through interconnections. This observation is in contrast to networks of one- or two-dimensional agents, and networks consisting of identical agents, where the ability of the system to reach consensus is determined by the graph Laplacian matrix alone [6], [7].

One immediate outcome of the above results concerns the design of communications between the filter nodes. In practice, it is often desirable to keep transmission of information between network nodes to a minimum, e.g., to improve the data throughput, save power, etc. The results of this paper indicate that, as far as the detectability of the entire system is concerned, the observability of the filters through interconnections must be an essential design consideration.

In regard to the role of communications, it is worthwhile to compare our conclusions with those in [8]. The approach undertaken in that reference is to construct interconnections to allow separation between the agents' closed loop control dynamics and their estimator error dynamics. In addition it achieves separation between the agents' estimator error dynamics. This leads to the conclusion that for the estimators to be able to converge, the system dynamics must be detectable from each individual node's measurements; see [8, Theorem 4]. In contrast, this paper considers the case where the estimator error dynamics remain coupled under communications. Coupling between the error dynamics allows us to show that the system can be detectable, even when the plant is not detectable from individual node's measurements.

The paper is organized as follows. In Section II we formulate the problem. The main results of the paper are given in Section III. In Section IV] an illustrative example is presented. 
Brief conclusions are given in Section $\nabla$, A conference version of this paper was presented at the 51st IEEE CDC [11].

Notation: Throughout the paper, $\mathbf{R}^{n}$ denotes the real Euclidean $n$-dimensional vector space. The symbol' denotes the transpose of a matrix or a vector. Ker $A$ denotes the null-space of a matrix $A . \mathbf{0}_{k} \triangleq\left[\begin{array}{lll}0 & \ldots & 0\end{array}\right]^{\prime} \in \mathbf{R}^{k}, \mathbf{1}_{k} \triangleq\left[\begin{array}{lll}1 & \ldots & 1\end{array}\right]^{\prime} \in \mathbf{R}^{k}$, and $I_{k}$ and $\mathbf{0}_{n \times k}$ are the identity matrix and the zero matrix; we will omit the subscripts when this causes no ambiguity. The symbol $\otimes$ denotes the Kronecker product of matrices, or the tensor product of two vector spaces. $\prod_{l=1}^{N} \mathscr{P}_{l}$ will denote the Cartesian product of $N$ vector spaces $\mathscr{P}_{1}, \ldots, \mathscr{P}_{N} \cdot \operatorname{dim} \mathscr{X}$ is the dimension of a finite dimensional vector space $\mathscr{X}$. $\operatorname{diag}\left[P_{1}, \ldots, P_{N}\right]$ denotes the block-diagonal matrix, whose diagonal blocks are $P_{1}, \ldots, P_{N}$.

\section{THE PROBLEM FORMULATION}

\section{A. Graph theory}

Consider a filter network with $N$ nodes and a directed graph topology $\mathbf{G}=(\mathbf{V}, \mathbf{E}) ; \mathbf{V}=\{1,2, \ldots, N\}, \mathbf{E} \subset \mathbf{V} \times \mathbf{V}$ are the set of vertices and the set of edges, respectively. The notation $(j, i)$ will denote the edge of the graph originating at node $j$ and ending at node $i$. In accordance with a common convention [6], we consider graphs without self-loops, i.e., $(i, i) \notin \mathbf{E}$. However, each node is assumed to have complete information about its filter and measurements.

For each $i \in \mathbf{V}$, let $\mathbf{V}_{i}=\{j:(j, i) \in \mathbf{E}\}$ be the set of nodes supplying information to node $i$, known as the neighbourhood of $i$. The cardinality of $\mathbf{V}_{i}$, known as the indegree of node $i$, is denoted $p_{i}$; i.e., $p_{i}$ is equal to the number of incoming edges for node $i$. Node $i$ of a digraph is said to be reachable from node $j$ if there exists a directed path originating at $j$ and ending at $i$. The graph is weakly connected if any two nodes are connected by an undirected path; it is strongly connected if its every node is reachable from any other node.

Let $\mathbf{A}=\left[\mathbf{a}_{i j}\right]_{i, j=1}^{N}$ be the adjacency matrix of the digraph $\mathbf{G}$, i.e., $\mathbf{a}_{i j}=1$ if $(j, i) \in \mathbf{E}$, otherwise $\mathbf{a}_{i j}=0$. Throughout the paper, $\mathscr{L}$ will denote the $N \times N$ Laplacian matrix of the $\operatorname{graph} \mathbf{G}, \mathscr{L}=\operatorname{diag}\left[p_{1}, \ldots, p_{N}\right]-\mathbf{A}$. Since $\mathbf{G}$ has no self-loops, entries within each row of $\mathscr{L}$ add up to 0 . Hence 0 is the eigenvalue of $\mathscr{L}$, and $\mathbf{1}_{N}$ is the corresponding eigenvector. This eigenvalue has multiplicity one if and only if the interconnection graph has a spanning tree [7].

\section{B. Motivating example: distributed estimation for SLAM}

To motivate the distributed detectability problem in this paper consider a simplified 2-D SLAM problem in which two robotic vehicles are required to determine the position of a static landmark as well as the position of each other. One of the most basic models for such SLAM system is

$$
\begin{aligned}
& {\left[\begin{array}{l}
\dot{x}_{1}^{(1)} \\
\dot{x}_{2}^{(1)}
\end{array}\right]=\left[\begin{array}{l}
\xi_{x}^{(1)} \\
\xi_{y}^{(1)}
\end{array}\right], \quad\left[\begin{array}{l}
\dot{x}_{1}^{(2)} \\
\dot{x}_{2}^{(2)}
\end{array}\right]=\left[\begin{array}{l}
\xi_{x}^{(2)} \\
\xi_{y}^{(2)}
\end{array}\right],} \\
& {\left[\begin{array}{c}
\dot{x}_{1}^{L} \\
\dot{x}_{2}^{L}
\end{array}\right]=\left[\begin{array}{l}
0 \\
0
\end{array}\right],}
\end{aligned}
$$

where $x_{1}^{(1)}, x_{2}^{(1)}, x_{1}^{(2)}, x_{2}^{(2)}, x_{1}^{L}, x_{2}^{L}$ are coordinates of the robots 1,2 and the landmark, respectively; $\xi_{x, 1}, \xi_{y, 1}, \xi_{x, 2}, \xi_{y, 2}$ are velocity inputs for the vehicles. The matrix form of (1) is

$$
\dot{x}=A x+B_{2} \xi(t), \quad x(0)=x_{0},
$$

where $x=\left(x_{1}^{(1)}, x_{2}^{(1)}, x_{1}^{(2)}, x_{2}^{(2)}, x_{1}^{L}, x_{2}^{L}\right)^{\prime}$ is the state vector, and $\xi(t)=\left(\xi_{x, 1}, \xi_{y, 1}, \xi_{x, 2}, \xi_{y, 2}\right)^{\prime}$. Also in this example $A=\mathbf{0}_{6 \times 6}, B_{2}=\left[\begin{array}{ll}I_{4} & \mathbf{0}_{4 \times 2}\end{array}\right]^{\prime}$. Measurements used by each robot consist of relative robot-to-landmark measurements and measurements of its own position (e.g., using GPS):

$$
y_{i}(t)=C_{i} x(t)+D_{i} \xi(t)+\bar{D}_{i} \xi^{i}(t),
$$

where $\xi^{1}(t), \quad \xi^{2}(t)$ are measurement noises, $C_{1}=$ $\left[\begin{array}{rrr}-I_{2} & \mathbf{0}_{2 \times 2} & I_{2} \\ I_{2} & \mathbf{0}_{2 \times 2} & \mathbf{0}_{2 \times 2}\end{array}\right], C_{2}=\left[\begin{array}{rrr}\mathbf{0}_{2 \times 2} & -I_{2} & I_{2} \\ \mathbf{0}_{2 \times 2} & I_{2} & \mathbf{0}_{2 \times 2}\end{array}\right], D_{1,2}=\mathbf{0}_{4 \times 4}$, $\bar{D}_{1,2}=I_{4}$. With this notation, the SLAM problem reduces to a state estimation problem in which each robot uses measurements (3) to estimate the state $x$ of the system (2). However, it is easy to see that each of the matrix pairs $\left(C_{1}, A\right)$, $\left(C_{2}, A\right)$ have undetectable modes, thus rendering standard state estimation approaches infeasible.

A further analysis reveals that the undetectable subspace of $\left(C_{1}, A\right)$ consists of vectors $\left[\begin{array}{llllll}0 & 0 & a & b & 0 & 0\end{array}\right]^{\prime}$ which indicates that the position of robot 2 is not observable by robot 1 . This problem will not arise if the robots avail each other of their measurements (since the pair $\left(\left[\begin{array}{ll}C_{1}^{\prime} & C_{2}^{\prime}\end{array}\right]^{\prime}, A\right)$ is observable). Another solution is to allow robot 2 to share the estimate of its own position with robot 1 , and vice versa. This leads us to introduce the following distributed SLAM estimator,

$$
\begin{aligned}
& \dot{\hat{x}}_{1}=A \hat{x}_{1}+L_{1}\left(y_{1}-C_{1} \hat{x}_{1}\right)+K_{1}\left(\hat{z}_{2}-H_{1} \hat{x}_{1}\right), \\
& \dot{\hat{x}}_{2}=A \hat{x}_{2}+L_{2}\left(y_{2}-C_{2} \hat{x}_{2}\right)+K_{2}\left(\hat{z}_{1}-H_{2} \hat{x}_{2}\right) .
\end{aligned}
$$

Here, $\hat{x}_{1}, \hat{x}_{2}$ denote the estimates of the vector $x$ computed by robots 1,2 , and $\hat{z}_{1}=H_{2} \hat{x}_{1}, \hat{z}_{2}=H_{1} \hat{x}_{2}$ are the estimates of the robot 1 and 2 own positions, respectively, to be shared with the neighbour; $H_{1}=\left[\begin{array}{lll}\mathbf{0}_{2 \times 2} & I_{2} & \mathbf{0}_{2 \times 2}\end{array}\right], H_{2}=\left[\begin{array}{lll}I_{2} & \mathbf{0}_{2 \times 2} & \mathbf{0}_{2 \times 2}\end{array}\right]$.

Depending on the nature of $\xi, \xi^{i}$ and the performance objective, the estimators in (4) can be seen as Kalman filters or $H_{\infty}$ filters. In both cases, the filter design is facilitated by the fact that the pairs $\left(\left[C_{i}^{\prime} H_{i}\right]^{\prime}, A\right)$ are observable, and 0 is the only state shared by the undetectable subspace of $\left(C_{i}, A\right)$ and the observable subspace of $\left(H_{i}, A\right)$. We will show that this condition is necessary and (under additional assumptions) sufficient for detectability of a general class of distributed estimator networks similar to (4).

The interconnection matrices $H_{1}, H_{2}$ given here are not unique to guarantee detectability for the SLAM filter (4). For example, it is easy to check that using the 'weighted disagreements' $H\left(\hat{x}_{2}-\hat{x}_{1}\right), H\left(\hat{x}_{1}-\hat{x}_{2}\right)$ where $H=H_{1}+H_{2}$, instead of the 'innovations' $\hat{z}_{2}-H_{1} \hat{x}_{1}, \hat{z}_{1}-H_{2} \hat{x}_{2}$, will not affect the observability and convergence properties of the filter. In general, we will see that the analysis of the entire observer network and its implementation is considerably simpler if all the agents utilize the same matrix $H$ in their communication protocols, and the detectabilty of the network is naturally expressed in terms of detectability properties of each network component. However, efficient communication protocols of this form may not be so obvious to find. The results in this paper aim at aiding in the development of such protocols. 


\section{The distributed detectability problem}

Consider the state estimation problem for a general system of the form (2), using a network of filters connected according to the graph $\mathbf{G}$. In (2), $x \in \mathbf{R}^{n}$ is the state of the plant, and $\xi$ denotes a disturbance signal. The sensing node $i$ uses measurements of the plant given by (3); $\xi^{i}$ represents the measurement uncertainty or the measurement noise at this node, $C_{i}, D_{i}, \bar{D}_{i}$ are given matrices. Node $i$ computes its estimate of the state $x$, denoted $\hat{x}_{i} \in \mathbf{R}^{n}$, using the filter

$$
\begin{array}{r}
\dot{\hat{x}}_{i}=A \hat{x}_{i}+L_{i}\left(y_{i}(t)-C_{i} \hat{x}_{i}\right)+K_{i} \sum_{j \in \mathbf{V}_{i}}\left(H_{i} \hat{x}_{j}-H_{i} \hat{x}_{i}\right), \\
\hat{x}_{i}(0)=0,
\end{array}
$$

Here $H_{i}, i=1, \ldots, N$, are given matrices. The filter (5) is a general form observer. According to (5), each node computes its estimate of the plant state $x$ from its local measurements $y_{i}$ and the inputs $H_{i} \hat{x}_{j}$ received from its neighbours, and also communicates to the neighbours its outputs $H_{k} \hat{x}_{i}$. The term $H_{i}\left(\hat{x}_{j}-\hat{x}_{i}\right)$ reflects the desire of each filter node to track the plant by reaching consensus with its neighbours. The matrices $L_{i}, K_{i}$ are the gain coefficients of the filter. Depending on the nature of disturbances and performance objectives, these coefficients can be determined so that the observers (5) perform as distributed Kalman filters or distributed $H_{\infty}$ filters [9], [10].

In this paper we are not concerned with filter performance against disturbances of a particular nature. We are interested in necessary conditions for asymptotic convergence of every node estimator (5) to the plant in the noise-free environment, which is a natural requirement to ensure fidelity of the estimates. Formally, it amounts to the existence of output injection matrices $L_{i}, K_{i}, i=1, \ldots, N$, such that the interconnected system consisting of the error dynamics subsystems

$$
\dot{e}_{i}=\left(A-L_{i} C_{i}\right) e_{i}+K_{i} H_{i} \sum_{j \in \mathbf{V}_{i}}\left(e_{j}-e_{i}\right)
$$

is globally asymptotically stable; here $e_{i}=x-\hat{x}_{i}$ is the local estimation error at node $i$. Let $\bar{A}=I_{N} \otimes A$, $\bar{C}=\operatorname{diag}\left[C_{1}, \ldots, C_{N}\right]$, and $\bar{H}=\left[\bar{H}_{i j}\right]_{i, j=1, \ldots, N}$ where $\bar{H}_{i j}=p_{i} H_{i}$ if $j=i$, and $\bar{H}_{i j}=-\mathbf{a}_{i j} H_{i}$ if $j \neq i$, then this requirement amounts to the detectability of $\left(\left[\bar{C}^{\prime}, \bar{H}^{\prime}\right]^{\prime}, \bar{A}\right)$.

From now on, we will assume identical matrices $H_{i}$ for all filters (5), $H_{i}=H$. Then $\bar{H}=\mathscr{L} \otimes H$. The intuition behind this assumption is drawn from the example in Section II-B where the detectability of the network was not affected when we replaced communication protocol matrices for both agents with judiciously selected identical matrices. In mobile networks with varying topology using the same matrix $H$ may have some merits. E.g., this enables all agents to use the same communication protocol, irrespective their location.

In the next section, we relate detectability of $\left(\left[\bar{C}^{\prime}, \bar{H}^{\prime}\right]^{\prime}, \bar{A}\right)$ with detectability properties of $\left(C_{i}, A\right)$, observability of $(H, A)$, and properties of the graph Laplacian $\mathscr{L}$.

\section{The Main Results}

\section{A. Geometric conditions for distributed detectability}

First let us recall the definition of the undetectable subspace of a matrix pair $(G, F), F \in \mathbf{R}^{n \times n}, G \in \mathbf{R}^{m \times n}$. Let
$\alpha_{F}(s)$ denote the minimal polynomial of $F$, i.e., the monic polynomial of least degree such that $\alpha_{F}(F)=0$ [13], factored as $\alpha_{F}(s)=\alpha_{F}^{-}(s) \alpha_{F}^{+}(s)$; the zeros of $\alpha_{F}^{-}(s)$ and $\alpha_{F}^{+}(s)$ are in the open left and closed right half-planes of the complex plane, respectively. Note that $\operatorname{Ker} \alpha_{F}^{-}(F) \cap \operatorname{Ker} \alpha_{F}^{+}(F)=\{0\}$, and $\operatorname{Ker} \alpha_{F}^{-}(F)+\operatorname{Ker} \alpha_{F}^{+}(F)=\mathbf{R}^{n}$ [13]. The undetectable subspace of $(G, F)$ is the subspace $\bigcap_{l=1}^{n} \operatorname{Ker}\left(G F^{l-1}\right) \cap$ $\operatorname{Ker} \alpha_{F}^{+}(F)$ [2]. When $F$ is the state matrix $A$, the notation $O_{G}$ will refer to the observability matrix associated with $(G, A)$, $O_{G}=\left[G^{\prime}(G A)^{\prime} \ldots\left(G A^{n-1}\right)^{\prime}\right]^{\prime}$.

Consider the undetectable subspaces of $\left(C_{i}, A\right)$ and the unobservable subspace of $(H, A)$, which will be denoted $\mathscr{C}_{i}$, $\mathscr{O}_{H}$. Furthermore, let $\overline{\mathscr{O}}$ denote the unobservable subspace of $(\bar{H}, \bar{A}), \overline{\mathcal{O}} \triangleq \bigcap_{l=1}^{n N} \operatorname{Ker}\left(\bar{H} \bar{A}^{l-1}\right)$. The following general result shows that the large-scale system (6) is detectable if and only if every combination of undetectable states of the pairs $\left(C_{i}, A\right)$ forms an observable state of $(\bar{H}, \bar{A})$.

Lemma 1: $\left(\left[\bar{C}^{\prime}, \bar{H}^{\prime}\right]^{\prime}, \bar{A}\right)$ is detectable if and only if

$$
\overline{\mathscr{O}} \cap \prod_{i=1}^{N} \mathscr{C}_{i}=\{0\} .
$$

The following lemma will be used in the proof of Lemma 1 Lemma 2: Recall that $\bar{A}=I_{N} \otimes A$. The following holds

$$
\operatorname{Ker} \alpha_{\bar{A}}^{+}(\bar{A})=\left(\operatorname{Ker} \alpha_{A}^{+}(A)\right)^{N} .
$$

The proof of this lemma is based on the observation that $\alpha_{A}(s)$ is the minimal polynomial for $\bar{A}$, and also $\alpha_{\bar{A}}^{+}(s)=$ $\alpha_{A}^{+}(s)$.

Proof of Lemma [1 Using Theorem 65 [2, p.259], and the fact that $\operatorname{Ker}\left[\begin{array}{l}P \\ Q\end{array}\right]=\operatorname{Ker} P \cap \operatorname{Ker} Q$, the condition of detectability of $\left(\left[\bar{C}^{\prime}, \bar{H}^{\prime}\right]^{\prime}, \bar{A}\right)$ can be equivalently written as $\left(\bigcap_{l=1}^{n N} \operatorname{Ker}\left(\bar{C} \bar{A}^{l-1}\right)\right) \cap \operatorname{Ker} \alpha_{\bar{A}}^{+}(\bar{A}) \cap \overline{\mathscr{O}}=\{0\}$. Therefore to prove the lemma, we need to show that

$$
\left(\bigcap_{l=1}^{n N} \operatorname{Ker}\left(\bar{C} \bar{A}^{l-1}\right)\right) \cap \operatorname{Ker} \alpha_{\bar{A}}^{+}(\bar{A})=\prod_{i=1}^{N} \mathscr{C}_{i} .
$$

First, consider the set $\bigcap_{l=1}^{n N} \operatorname{Ker} \bar{C} \bar{A}^{l-1}$ and take an arbitrary vector $x$ in that set, partitioned as $x=\left[\begin{array}{lll}x_{1}^{\prime} & \ldots & x_{N}^{\prime}\end{array}\right]^{\prime}, x_{i} \in$ $\mathbf{R}^{n}$. Given that $\bar{C}$ and $\bar{A}$ are block diagonal, the condition $x \in \bigcap_{l=1}^{n N} \operatorname{Ker} \bar{C} \bar{A}^{l-1}$ is equivalent to $x_{i} \in \operatorname{Ker} C_{i} A^{l-1}$, for all $i=1, \ldots, N$ and all $l=1, \ldots, n N$. This implies $x_{i} \in$ Ker $O_{C_{i}}$ for all $i=1, \ldots, N$. Therefore, $\bigcap_{l=1}^{n N} \operatorname{Ker} \bar{C} \bar{A}^{l-1} \subseteq$ $\prod_{i=1}^{N} \operatorname{Ker} O_{C_{i}}$.

Conversely, take $y_{i} \in \operatorname{Ker} O_{C_{i}}$. Using the CayleyHamilton theorem, this implies that $y=\left[\begin{array}{lll}y_{1}^{\prime} & \ldots & y_{N}^{\prime}\end{array}\right]^{\prime} \in$ $\operatorname{Ker} \bar{C} \bar{A}^{l-1}$ for all $l=1, \ldots, n N$. This leads to the conclusion that $\prod_{i=1}^{N} \operatorname{Ker} O_{C_{i}} \subseteq \bigcap_{l=1}^{n N} \operatorname{Ker} \bar{C} \bar{A}^{l-1}$. Hence, $\bigcap_{l=1}^{n N} \operatorname{Ker} \bar{C} \bar{A}^{l-1}=\prod_{i=1}^{N} \operatorname{Ker} O_{C_{i}}$.

To complete the proof, we now refer to Lemma 2 , where we showed that $\operatorname{Ker} \alpha_{\bar{A}}^{+}(\bar{A})=\left(\operatorname{Ker} \alpha_{A}^{+}(A)\right)^{N}$. Since by definition, $\mathscr{C}_{i}=\operatorname{Ker} O_{C_{i}} \cap \operatorname{Ker} \alpha_{A}^{+}(A)$, then (9) follows, as required.

Remark 1: One can see from this proof that Lemma1 1 holds in a more general case where the matrices $H_{i}$ are not identical. 
Lemma 3: Recall that $\mathscr{O}$ is the unobservable subspace of the pair $(\bar{H}, \bar{A})$. The following holds

$$
\overline{\mathscr{O}}=(\operatorname{Ker} \mathscr{L}) \otimes \mathbf{R}^{n}+\left(\bigcap_{l=1}^{n} \operatorname{Ker}\left(H A^{l-1}\right)\right)^{N} .
$$

Proof: First we observe that $\overline{\mathscr{O}}=\operatorname{Ker}\left(\mathscr{L} \otimes O_{H}\right)$. Indeed, note that $(\mathscr{L} \otimes H)(I \otimes A)^{l-1}=\mathscr{L} \otimes\left(H A^{l-1}\right)$. Hence $\overline{\mathscr{O}}=$ $\operatorname{Ker}\left[(\mathscr{L} \otimes H)^{\prime}(\mathscr{L} \otimes(H A))^{\prime} \ldots\left(\mathscr{L} \otimes\left(H A^{n N-1}\right)\right)^{\prime}\right]^{\prime}$. This implies that $x=\left[\begin{array}{lll}x_{1}^{\prime} & \ldots & x_{N}^{\prime}\end{array}\right] \in \overline{\mathscr{O}}$ if and only if

$$
\sum_{j \in \mathbf{V}_{i}} H A^{l-1}\left(x_{i}-x_{j}\right)=0, \quad l=1, \ldots, n N .
$$

By the Hamilton-Caley theorem, for all $l \geq n$ one can find constants $a_{1, l}, \ldots, a_{n, l}$, such that $A^{\bar{l}} z=$ $\sum_{\nu=1}^{n} a_{\nu, l}\left(A^{\nu-1} z\right) \quad \forall z \in \mathbf{R}^{n}$. Using this general identity, we establish that for all $l \geq n$,

$$
\sum_{j \in \mathbf{V}_{i}} H A^{l}\left(x_{i}-x_{j}\right)=\sum_{\nu=1}^{n} a_{\nu, l}\left(\sum_{j \in \mathbf{V}_{i}} H A^{\nu-1}\left(x_{i}-x_{j}\right)\right) .
$$

Hence, 11 holds for all $l=1, \ldots, n N$ if and only if it holds for all $l=1, \ldots, n$. This proves that $\overline{\mathscr{O}}=\operatorname{Ker}\left(\mathscr{L} \otimes O_{H}\right)$.

Using this representation of $\overline{\mathscr{O}}$ and the fact that $\bigcap_{l=1}^{n} \operatorname{Ker}\left(H A^{l-1}\right)=\mathscr{O}_{H}$, the identity (10) can be re-written as

$$
\operatorname{Ker}\left(\mathscr{L} \otimes O_{H}\right)=(\operatorname{Ker} \mathscr{L}) \otimes \mathbf{R}^{n}+\left(\mathscr{O}_{H}\right)^{N} .
$$

To prove (12) we first show that $\operatorname{Ker} \mathscr{L} \otimes \mathbf{R}^{n}+\left(\mathscr{O}_{H}\right)^{N} \subseteq$ $\operatorname{Ker}\left(\mathscr{L} \otimes O_{H}\right)$. It suffices to check this for elements of the subspaces $(\operatorname{Ker} \mathscr{L}) \otimes \mathbf{R}^{n}$ and $\left(\mathscr{O}_{H}\right)^{N}$, separately. Every element of $(\operatorname{Ker} \mathscr{L}) \otimes \mathbf{R}^{n}$ is a vector of the form $b \otimes z$, where $b \in \operatorname{Ker} \mathscr{L}$, and $z \in \mathbf{R}^{n}$. Therefore $\left(\mathscr{L} \otimes O_{H}\right)(b \otimes z)=$ $\mathscr{L} b \otimes O_{H} z=0$. Also, choose arbitrary elements of $\mathscr{O}_{H}, h_{i}$, $i=1, \ldots, N$. Then $h=\left[h_{1}^{\prime} \ldots h_{N}^{\prime}\right]^{\prime} \in\left(\mathscr{O}_{H}\right)^{N}$, and

$$
\left(\mathscr{L} \otimes O_{H}\right) h=\left[\begin{array}{c}
\sum_{j \in \mathbf{V}_{1}} O_{H}\left(h_{1}-h_{j}\right) \\
\vdots \\
\sum_{j \in \mathbf{V}_{N}} O_{H}\left(h_{N}-h_{j}\right)
\end{array}\right]=0 .
$$

The inclusion $\operatorname{Ker} \mathscr{L} \otimes \mathbf{R}^{n}+\left(\mathscr{O}_{H}\right)^{N} \subseteq \operatorname{Ker}\left(\mathscr{L} \otimes O_{H}\right)$ then follows.

To prove that this inclusion is in fact the identity, and thus complete the proof of the lemma, we now show that the subspaces on both sides of the inclusion have the same dimension, that is

$$
\operatorname{dim}\left((\operatorname{Ker} \mathscr{L}) \otimes \mathbf{R}^{n}+\left(\mathscr{O}_{H}\right)^{N}\right)=\operatorname{dim} \operatorname{Ker}\left(\mathscr{L} \otimes O_{H}\right) .
$$

To prove this, let $d_{\mathscr{L}}, d_{\mathscr{O}}$ be the dimensions of the spaces $\operatorname{Ker} \mathscr{L}, \mathscr{O}_{H}$, respectively. The following identity holds [13]

$$
\begin{aligned}
& \operatorname{dim}\left((\operatorname{Ker} \mathscr{L}) \otimes \mathbf{R}^{n}+\left(\mathscr{O}_{H}\right)^{N}\right) \\
& \quad=n d_{\mathscr{L}}+N d_{\mathscr{O}}-\operatorname{dim}\left(\left((\operatorname{Ker} \mathscr{L}) \otimes \mathbf{R}^{n}\right) \cap\left(\mathscr{O}_{H}\right)^{N}\right) .
\end{aligned}
$$

To find the last term in the above equation, observe that a nonzero $x$ belongs to $\left((\operatorname{Ker} \mathscr{L}) \otimes \mathbf{R}^{n}\right) \cap\left(\mathscr{O}_{H}\right)^{N}$ if and only if it admits the decomposition $x=\left[b_{1} z^{\prime} \ldots b_{N} z^{\prime}\right]^{\prime}$ for some $z \in \mathbf{R}^{n}, z \neq 0$ and $b=\left[b_{1} \ldots b_{N}\right]^{\prime} \in \operatorname{Ker} \mathscr{L}, b \neq 0$, and also $b_{i} O_{H} z=0$ for all $i=1, \ldots, N$. Since $b \neq 0$, this implies that $z \in \mathscr{O}_{H}$. Hence, $\left((\operatorname{Ker} \mathscr{L}) \otimes \mathbf{R}^{n}\right) \cap\left(\mathscr{O}_{H}\right)^{N}=(\operatorname{Ker} \mathscr{L}) \otimes \mathscr{O}_{H}$. Thus, we conclude that $\operatorname{dim}\left((\operatorname{Ker} \mathscr{L}) \otimes \mathbf{R}^{n}+\left(\mathscr{O}_{H}\right)^{N}\right)=$ $n d_{\mathscr{L}}+\left(N-d_{\mathscr{L}}\right) d_{\mathscr{O}}$.

On the other hand, $\operatorname{dim} \operatorname{Ker}\left(\mathscr{L} \otimes O_{H}\right)=n N-(N-$ $\left.d_{\mathscr{L}}\right)\left(n-d_{\mathscr{O}}\right)=n d_{\mathscr{L}}+\left(N-d_{\mathscr{L}}\right) d_{\mathscr{O}}$. Therefore, (13) holds. This shows that the statement of the lemma holds true.

Our first main result, given below, presents necessary conditions for the detectability of the pair $\left(\left[\bar{C}^{\prime}, \bar{H}^{\prime}\right]^{\prime}, \bar{A}\right)$.

Theorem 1: Suppose the pair $\left(\left[\bar{C}^{\prime}, \bar{H}^{\prime}\right]^{\prime}, \bar{A}\right)$ is detectable. Then, the following statements hold:

(i) $\bigcap_{i=1}^{N} \mathscr{C}_{i}=\{0\}$;

(ii) $\mathscr{O}_{H} \cap \mathscr{C}_{i}=\{0\}$ for all $i=1, \ldots, N$;

(iii) $\operatorname{rank} O_{H} \geq \max _{i} \operatorname{dim} \mathscr{C}_{i}$.

Proof: (i) Suppose $z \in \bigcap_{i=1}^{N} \mathscr{C}_{i}$. Then it follows from Lemma 3 that $\mathbf{1}_{N} \otimes z \in(\operatorname{Ker} \mathscr{L}) \otimes \mathbf{R}^{n} \subseteq \overline{\mathscr{O}}$. Also by definition, $\mathbf{1}_{N} \otimes z \in \prod_{i=1}^{N} \mathscr{C}_{i}$. Hence, it follows from Lemma 1 that $\mathbf{1}_{N} \otimes z=0$ which implies $z=0$. This proves claim (i).

(ii) Suppose $y_{i} \in \mathscr{O}_{H} \cap \mathscr{C}_{i}$ and consider the vector $y=$ $\left[\begin{array}{llll}\delta_{1 i} & \delta_{2 i} & \ldots & \delta_{N i}\end{array}\right]^{\prime} \otimes y_{i}$, where $\delta_{s i}$ is the Kronecker symbol. By definition, $y \in\left(\mathscr{O}_{H}\right)^{N} \subseteq \overline{\mathscr{O}}$ and $y \in \prod_{i=1}^{N} \mathscr{C}_{i}$. Hence, by Lemma 1, $y=0$. This implies $y_{i}=0$, which proves claim (ii).

(iii) From (ii), $n \geq \operatorname{dim}\left(\mathscr{O}_{H}+\mathscr{C}_{i}\right)=\operatorname{dim} \mathscr{O}_{H}+\operatorname{dim} \mathscr{C}_{i}$. Since $\operatorname{rank} O_{H}=n-\operatorname{dim} \mathscr{O}_{H}$, the claim follows.

Statement (ii) of Theorem 1 means that for the distributed output injection problem stated in Section $\amalg$ to have a solution, every undetectable state of $\left(C_{i}, A\right)$ must necessarily be an observable state of $(H, A)$. Also, every unobservable state of $(H, A)$ must be a detectable state of one of the pairs $\left(C_{i}, A\right)$.

\section{B. Detectability over graphs spanned by trees}

Our second main result shows that the conditions given in statements (i) and (ii) of Theorem 1 are in fact, sufficient for the detectability of $\left(\left[\bar{C}^{\prime} \bar{H}^{\prime}\right]^{\prime}, \bar{A}\right)$, provided the graph Laplacian matrix has a zero eigenvalue of multiplicity one. As is well known, this condition holds if and only if the interconnection graph has a spanning tree [7]. The result given in Theorem 2 below presents conditions on the graph connectivity, which ensure that each node observer receives a necessary complementary information through the interconnections.

Theorem 2: Suppose the interconnection graph $\mathscr{G}$ has a spanning tree. If the conditions given in statements (i) and (ii) of Theorem 1 hold, then the pair $\left(\left[\bar{C}^{\prime} \bar{H}^{\prime}\right]^{\prime}, \bar{A}\right)$ is detectable.

Proof: Since $\mathscr{G}$ has a spanning tree, then the geometric multiplicity of the zero eigenvalue of the graph Laplacian matrix $\mathscr{L}$ is equal to 1 . Hence the eigenvector $\mathbf{1}_{N}$ is the only eigenvector (modulo scaling) corresponding to the zero eigenvalue of $\mathscr{L}$. From this fact and Lemma 3 , it follows that every element of $\overline{\mathscr{O}}$ has the form $\left[\left(z+h_{1}\right)^{\prime} \ldots\left(z+h_{N}\right)^{\prime}\right]^{\prime}$, where $h_{1}, \ldots, h_{N} \in \mathscr{O}_{H}$, and $z$ is an arbitrary vector $z \in \mathbf{R}^{n}$.

Suppose there exists a vector of the above form which also belongs to $\prod_{j=1}^{N} \mathscr{C}_{j}$. This implies the existence of $z \in \mathbf{R}^{n}$, and $h_{1}, \ldots, h_{N} \in \mathscr{O}_{H}$ such that $\forall i=1, \ldots, N$,

$$
O_{C_{i}} z=-O_{C_{i}} h_{i}, \quad \alpha_{A}^{+}(A) z=-\alpha_{A}^{+}(A) h_{i} .
$$

However, property (i) of Theorem 1 means that the matrix $\left[\begin{array}{llllll}O_{C_{1}}^{\prime} & \ldots & O_{C_{N}}^{\prime} & \alpha_{A}^{+}(A)^{\prime} & \ldots & \alpha_{A}^{+}(A)^{\prime}\end{array}\right]^{\prime}$ of the system (14) has 
full row rank. Therefore, if $z \in \mathbf{R}^{n}$, and $h_{1}, \ldots, h_{N} \in \mathscr{O}_{H}$ satisfy (14), then $z$ must be a linear combination of the vectors $h_{1} \ldots, h_{N}$. Thus, $z \in \mathscr{O}_{H}$ and also $z+h_{i} \in \mathscr{O}_{H}$ for all $i$. Using property (ii) of Theorem 1 we conclude that $z+h_{i}=0$ for all $i=1, \ldots, N$. Hence (7) holds. According to Lemma 1 this means that the pair $\left(\left[\begin{array}{ll}\bar{C}^{\prime} & \bar{H}^{\prime}\end{array}\right]^{\prime}, \bar{A}\right)$ is detectable.

We now specialize Theorem 2 to some special distributed observer topologies commonly considered in the literature. The result of Corollary 2 applies in the situation where the root node of the graph plays the role of the leader who estimates the plant and then passes its estimates to other nodes [5]. On the contrary, Corollary 1 applies to leaderless observer networks such as the networks connected over balanced strongly connected graphs considered in [10].

Corollary 1: Suppose $(H, A)$ is observable. Also, suppose the interconnection graph $\mathscr{L}$ has a spanning tree. If property (i) of Theorem 1 holds, then the pair $\left(\left[\begin{array}{ll}\bar{C}^{\prime} & \bar{H}^{\prime}\end{array}\right]^{\prime}, \bar{A}\right)$ is detectable.

An immediate implication of Corollary 1 is that the observability of the pair $(H, A)$ must be an essential design consideration when choosing a suitable matrix $H$ for information exchange between the nodes in a leaderless network.

Corollary 2: Suppose the graph $\mathbf{G}$ has a spanning tree, with node $i$ being the root node of the tree. Also, suppose $\left(C_{i}, A\right)$ is detectable at the root node. If property (ii) of Theorem 1 holds, then the pair $\left(\left[\begin{array}{ll}\bar{C}^{\prime} & \bar{H}^{\prime}\end{array}\right]^{\prime}, \bar{A}\right)$ is detectable.

\section{Detectability over graphs which are not spanned by a tree}

We now restrict attention to weakly connected graphs which fail to satisfy the connectivity assumptions of Section [II-B]. We show that in this case the system (6) is stabilizable via output injection if and only if certain observer clusters within the system have this property.

To characterize these clusters of observers, we first discuss the relation between the structure of the interconnection graph and the multiplicity of the zero eigenvalue of $\mathscr{L}$. The classical result in the graph theory states that the multiplicity of the zero eigenvalue of the Laplace matrix of an undirected graph is equal to the number of connected components of the graph. Recently, this result was extended to directed graphs [1], [3]. It was shown in these references that the multiplicity of the zero eigenvalue of $\mathscr{L}$ is equal to the number of maximal reachable subgraphs within the graph. To present these results, some terminology from [3] is needed. For any vertex $j$, the reachable subgraph of $j, \mathbf{R}(j)$, is defined to be the vertex subgraph containing node $j$ and all nodes reachable from $j$. A vertex subgraph $\mathbf{R}$ is a reach if it is a maximal reachable subgraph; i.e., if $\mathbf{R}=\mathbf{R}(i)$ for some $i$ and there is no $j \neq i$ such that $\mathbf{R}(i) \subset \mathbf{R}(j)$. A graph may consist of several reaches. For each reach $\mathbf{R}_{\mathbf{s}}$, the exclusive part of $\mathbf{R}_{s}$ is the vertex subgraph $\mathbf{P}_{s}=\mathbf{R}_{s} \backslash \cup_{r \neq s} \mathbf{R}_{r}$. The common part of $\mathbf{R}_{s}$ is the vertex subgraph $\mathbf{Q}_{s}=\mathbf{R}_{s} \backslash \mathbf{P}_{s}$.

It follows from these definitions that reaches have no outgoing edges. The estimators within a reach $\mathbf{R}_{\mathrm{s}}$ do not share information with estimators at nodes $j \notin \mathbf{R}_{\mathbf{s}}$ but can receive

\footnotetext{
${ }^{1}$ If the graph is disconnected, the estimation problem decouples into separate estimation problems [10 Proposition 1].
}

information from these nodes. On the other hand, the observers at nodes $i \in \mathbf{P}_{\mathbf{s}}$ do not receive information from nodes $j \notin \mathbf{P}_{\mathbf{s}}$ since by definition $i \in \mathbf{P}_{\mathbf{s}}$ is not reachable from $j \notin \mathbf{P}_{\mathbf{s}}$.

Lemma 4 (Corollary 4.2, [3]): The algebraic and geometric multiplicity of the eigenvalue 0 of $\mathscr{L}$ is equal to the number of reaches in the graph G. Furthermore, Ker $\mathscr{L}$ has a basis $b^{1}, \ldots, b^{k}$ whose elements satisfy the conditions:

(i) $b_{i}^{s}=0$ for $i \in \mathbf{G} \backslash \mathbf{R}_{s}, s=1, \ldots, k$;

(ii) $b_{i}^{s}=1$ for $i \in \mathbf{P}_{s}, s=1, \ldots, k$;

(iii) $0<b_{i}^{s}<1$ for $i \in \mathbf{Q}_{s}, s=1, \ldots, k$;

(iv) $\sum_{s=1}^{k} b^{s}=\mathbf{1}_{N}$.

Theorem 3.2 in [3] shows that by permuting rows and columns, $\mathscr{L}$ can be represented as

$$
\mathscr{L}=\left[\begin{array}{cccc}
\mathscr{L}_{1} & \ldots & 0 & 0 \\
0 & \ddots & 0 & 0 \\
0 & \ldots & \mathscr{L}_{k} & 0 \\
F_{1} & \ldots & F_{k} & R
\end{array}\right]
$$

where the first $k$ rows of blocks correspond to exclusive subgraphs $\mathbf{P}_{s} \subset \mathbf{G}$, and the remaining rows correspond to the vertices from $\cup_{s=1}^{k} \mathbf{Q}_{s}$. Since exclusive subgraphs $\mathbf{P}_{s}$ are not reachable from the nodes outside $\mathbf{P}_{s}$, each matrix $\mathscr{L}_{s}$, $s=1, \ldots, k$, is a Laplacian matrix of the corresponding subgraph $\mathbf{P}_{s}$, and its zero eigenvalue has multiplicity 1 . Also, $R$ is shown to be invertible. In accordance with this partition, after the permutation the vectors $b^{s}$ have the form $b^{s}=\left[\begin{array}{lll}\mathbf{0}_{l_{1}+\ldots+l_{s-1}}^{\prime} & \mathbf{1}_{l_{s}}^{\prime} & \mathbf{0}_{l_{s+1}+\ldots+l_{k}}^{\prime}\left(f^{s}\right)^{\prime}\end{array}\right]^{\prime}$, where $l_{q}=$ $\operatorname{dim} \mathscr{L}_{q}$ is the cardinality of the vertex set of $\mathbf{P}_{q}$. Also, $f^{s}=-R^{-1} F_{s} \mathbf{1}_{l_{s}} \in \mathbf{R}^{r}, r$ being the cardinality of the vertex set of $\cup_{s=1}^{k} \mathbf{Q}_{s}$. From Lemma 4, the vector $f^{s}$ can be further partitioned $f^{s}=\left[\left(f_{1}^{s}\right)^{\prime} \ldots\left(f_{k}^{s}\right)^{\prime}\right]^{\prime}$, where $f_{q}^{s}$ designates the component corresponding to the nodes of $\mathbf{Q}_{q}$ after the permutation. Therefore, $f_{q}^{s}=0$ for $q \neq s$, and all the entries of $f_{s}^{s}=\left[\begin{array}{llll}f_{s, 1}^{s} & \ldots & f_{s, r_{s}}^{s}\end{array}\right]^{\prime}$ corresponding to the vertices in $\mathbf{Q}_{s}$ satisfy $0<f_{s, i}^{s}<1 ; r_{s}$ denotes the cardinality of the vertex set of $\mathbf{Q}_{s}$.

Theorem 3: Suppose the pair $\left(\left[\bar{C}^{\prime}, \bar{H}^{\prime}\right]^{\prime}, \bar{A}\right)$ is detectable. Then, for every reach $\mathbf{R}_{s} \subset \mathbf{G}$ :

(i) $\bigcap_{i \in \mathbf{R}_{s}} \mathscr{C}_{i}=\{0\}$;

(ii) $\mathscr{O}_{H} \cap \mathscr{C}_{i}=\{0\}$ for all $i \in \mathbf{R}_{s}$.

Proof: Statement (ii) follows from Theorem 1 Now suppose that there exists a reach which fails to satisfy condition (i). Without loss of generality, take $\mathbf{R}_{1}$ to be this reach, with the exclusive part $\mathbf{P}_{1}$, and the common part $\mathbf{Q}_{1}$. Our assumption means that there exists $z \in \mathbf{R}^{n}$ such that $z \neq 0$ and $z \in\left(\bigcap_{i \in \mathbf{P}_{1}} \mathscr{C}_{i}\right) \cap\left(\bigcap_{i \in \mathbf{Q}_{1}} \mathscr{C}_{i}\right)$. Note that this implies $z \in$ $\bigcap_{i \in \mathbf{P}_{1}} \operatorname{Ker} O_{C_{i}}, z \in \bigcap_{i \in \mathbf{Q}_{1}} \operatorname{Ker} O_{C_{i}}$, and $z \in \operatorname{Ker} \alpha_{A}^{+}(A)$.

Consider the vector $y=b_{1} \otimes z \in \mathbf{R}^{n N}$. From Lemma 3 , $y \in \overline{\mathscr{O}}$, and $y \neq 0$ since $z \neq 0$. We now show that $y \in$ $\prod_{i=1}^{N} \mathscr{C}_{i}$. According to the discussion preceding the theorem, this vector can be partitioned as follows $y=\left[y_{1}^{\prime} \ldots y_{N}^{\prime}\right]^{\prime}$, where $y_{i}=z$ for $i \in \mathbf{P}_{1}, y_{i}=f_{1, i}^{1} z$ for $i \in \mathbf{Q}_{1}$, and $y_{i}=0$ for $i \in \mathbf{V} \backslash \mathbf{R}_{1}$. Therefore, for every node $i \in \mathbf{V} \backslash \mathbf{R}_{\mathbf{1}}$, we have $O_{C_{i}} y_{i}=0$. Also, for $i \in \mathbf{P}_{1}, O_{C_{i}} y_{i}=O_{C_{i}} z=0$ since $z \in \bigcap_{i \in \mathbf{P}_{1}} \operatorname{Ker} O_{C_{i}}$. Similarly, $O_{C_{i}} y_{i}=0$ for $i \in \mathbf{Q}_{1}$. Since $z \in \operatorname{Ker} \alpha_{A}^{+}(A)$, then $y_{i} \in \operatorname{Ker} \alpha_{A}^{+}(A)$. Thus, $y_{i} \in \mathscr{C}_{i} \forall i$. 
We have shown that $y \in \overline{\mathscr{O}} \cap \prod_{i=1}^{N} \mathscr{C}_{i}$. This leads to a contradiction with the condition that $\left(\left[\bar{C}^{\prime}, \bar{H}^{\prime}\right]^{\prime}, \bar{A}\right)$ is detectable; see (7). This proves the statement of the theorem.

Theorem 4: Suppose the pair $(H, A)$ is observable. If for every reach $\mathbf{R}$ in $\mathbf{G}, \bigcap_{i \in \mathbf{R}} \mathscr{C}_{i}=\{0\}$, then the pair $\left(\left[\bar{C}^{\prime}, \bar{H}^{\prime}\right]^{\prime}, \bar{A}\right)$ is detectable.

Proof: Suppose $\left(\left[\bar{C}^{\prime}, \bar{H}^{\prime}\right]^{\prime}, \bar{A}\right)$ is not detectable, and therefore there exists a nonzero vector $y \in \overline{\mathscr{O}}_{H} \cap \prod_{i=1}^{N} \mathscr{C}_{i}$. From Lemma 3 since the pair $(H, A)$ is observable, then $\overline{\mathscr{O}}_{H}=\operatorname{Ker} \mathscr{L} \otimes \mathbf{R}^{n}$. Hence, the vector $y$ can be represented as $y=b \otimes z$, where $z \in \mathbf{R}^{n}$, and $b=\sum_{s=1}^{k} c_{s} b^{s} ; c_{1}, \ldots, c_{k}$ are scalar constants. Furthermore, using Lemma 4, we have $b_{i}=c_{s}$ if $i \in \mathbf{P}_{s}, b_{i}=c_{s} f_{s, i}^{s}$ if $i \in \mathbf{Q}_{s}$, and $b_{i}=0$ otherwise. Since $y \neq 0$, this implies that in the representation $y=b \otimes z$, the vectors $z, b$ are nonzero. It further follows from the condition $b \neq 0$ that for at least one $s \in\{1, \ldots, k\}$, $c_{s} \neq 0$ and $c_{s} f_{s, i}^{s} \neq 0$ for all $i \in \mathbf{Q}_{s}$. Since the condition $y \in \prod_{i=1}^{N} \mathscr{C}_{i}$ implies $c_{s} z \in \operatorname{Ker} \alpha_{A}^{+}(A), c_{s} O_{C_{i}} z=0$ for $i \in \mathbf{P}_{s}$ and $c_{s} f_{s, i}^{s} O_{C_{i}} z=0$ for $i \in \mathbf{Q}_{s}$, this leads to the conclusion that $z \in \cap_{i \in \mathbf{R}_{s}} \mathscr{C}_{i}$, which contradicts the condition $\cap_{i \in \mathbf{R}_{s}} \mathscr{C}_{i}=\{0\}$. Hence $\left(\left[\bar{C}^{\prime}, \bar{H}^{\prime}\right]^{\prime}, \bar{A}\right)$ must be detectable.

Remark 2: Since a digraph spanned by a tree is a reach, the result of Corollary 1 can be seen as a special case of Theorem 4

\section{EXAMPLE}

In this section, we revisit Example 1 presented in [10]. The state equation of the reference plant in that example is 6dimensional and is governed by the $6 \times 6$ state matrix

$$
A=\left[\begin{array}{rrrrrr}
0.3775 & 0 & 0 & 0 & 0 & 0 \\
0.2959 & 0.3510 & 0 & 0 & 0 & 0 \\
1.4751 & 0.6232 & 1.0078 & 0 & 0 & 0 \\
0.2340 & 0 & 0 & 0.5596 & 0 & 0 \\
0 & 0 & 0 & 0.4437 & 1.1878 & -0.0215 \\
0 & 0 & 0 & 0 & 2.2023 & 1.0039
\end{array}\right] .
$$

The plant is observed by the network consisting of six $H_{\infty}$ filters of the form (5) connected in the topology of a directed ring. The 1st filter measures the 1 st and the 2 nd coordinates of the state vector, the 2 nd filter measures the 2 nd and the $3 \mathrm{rd}$ coordinates, etc, with the last filter taking measurements of the 6th and the 1st coordinates. In particular, $C_{2}=\left[\begin{array}{lll}\mathbf{0}_{2} & I_{2} & \mathbf{0}_{2 \times 3}\end{array}\right]$, $C_{4}=\left[\begin{array}{lll}\mathbf{0}_{2 \times 3} & I_{2} & \mathbf{0}_{2}\end{array}\right]$.

It can be directly verified that all eigenvalues of $A$ are in the right half-plane, hence at every node of the network, the unobservable modes of $A$ are not detectable. That is, $\mathscr{C}_{i}=\operatorname{Ker} O_{C_{i}}$. Furthermore, $\operatorname{Ker} O_{C_{2}}$ is spanned by the vectors $d_{4}, d_{5}$, and $d_{6}$, while $\operatorname{Ker} O_{C_{4}}$ is spanned by $d_{2}, d_{3}$; here $d_{i}$ is the $i$ th vector in the canonical orthogonal basis in $\mathbf{R}^{n}$. Hence, $\cap_{i=1}^{6} \mathscr{C}_{i}=0$. Also, $H=I_{6}$ in Example 1 of [10]. This guarantees that $(H, A)$ is observable. Finally, the 6node directed ring has a spanning tree. Thus, we have verified all conditions of Corollary 1 . According to Corollary 1 , the

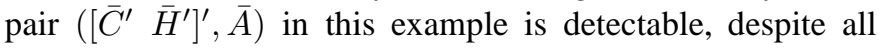
the individual pairs $\left(C_{i}, A\right)$ having nontrivial undetectable subspaces.

To confirm this finding, the detectability of the matrix pair $\left(\left[\bar{C}^{\prime}, \bar{H}^{\prime}\right], \bar{A}\right)$ was verified directly using Matlab. Also in [10], a set of stabilizing output injection gains was found and the stability of the system (6) was verified directly, thus confirming our conclusion based on Corollary 1.

It follows from Corollary 1 that the detectability of $\left(\left[\bar{C}^{\prime}, \bar{H}^{\prime}\right], \bar{A}\right)$ will be preserved even if the filters transmit, e.g., only the third and fifth coordinates of their respective estimate vectors, that is, if instead of $H=I_{6}$, we take $H=\left[\begin{array}{llllll}0 & 0 & 1 & 0 & 0 & 0 \\ 0 & 0 & 0 & 0 & 1 & 0\end{array}\right]$. With this $H,(H, A)$ is observable, and Corollary 1 is still applicable. This creates a potential for reducing the amount of information transmitted by the nodes, since only two coordinates of the estimate vectors $\hat{x}_{j}$ need to be transmitted instead of all six coordinates. However, if the filters transmit the 2nd and the 5th coordinates of $\hat{x}_{j}$, the pair $(H, A)$ will not be observable and the system cannot be guaranteed to be detectable. In fact, one can check directly that the corresponding pair $\left(\left[\bar{C}^{\prime}, \bar{H}^{\prime}\right], \bar{A}\right)$ is not detectable. Therefore, the distributed filter of the form (5) cannot be constructed in this case.

\section{CONCLUSIONS}

The paper presents necessary and sufficient conditions for detectability of a linear plant via a network of state estimators. We showed that the detectability of the entire system can be ascertained from the detectability properties of the filters' pairs $\left(C_{i}, A\right)$, along with the matching properties of interconnections. Our results complement the existing results on distributed consensus-based estimation by elucidating the relationship between the network topology and detectability/observability properties of the plant and filters. Future work will investigate a similar relationship between the network topology and controllability of multi-agent systems.

\section{REFERENCES}

[1] R. Agaev and P. Chebotarev. On the spectra of nonsymmetric Laplacian matrices. Linear Algebra and its Applications, 399:157 - 168, 2005.

[2] F.M. Callier and C.A. Desoer. Linear system theory. Springer, 1991.

[3] J. S. Caughman and J. J. P. Veerman. Kernels of directed graph Laplacians. The Electronic Journal of Combinatorics, 13, 2006. \#R39.

[4] G.P. Huang, A.I. Mourikis, and S.I. Roumeliotis. Analysis and improvement of the consistency of extended Kalman filter based SLAM. In IEEE Int. Conf. Robotics and Automation, 2008, pp. 473-479, 2008.

[5] Z. Li, Z. Duan, G. Chen, and L. Huang. Consensus of multiagent systems and synchronization of complex networks: A unified viewpoint. IEEE Trans. Circuits Syst. I: Regular Papers, 57:213-224, 2010.

[6] R. Olfati-Saber and R. M. Murray. Consensus problems in networks of agents with switching topology and time-delays. IEEE Trans. Automat. Contr., 49:1520-1533, 2004.

[7] W. Ren and R. W. Beard. Consensus seeking in multiagent systems under dynamically changing interaction topologies. IEEE Trans. Automat. Contr., 50(5):655-661, 2005.

[8] R. Smith and F. Hadaegh. Closed-loop dynamics of cooperative vehicle formations with parallel estimators and communication. IEEE Trans. Automat. Contr., 52(8):1404-1414, 2007.

[9] M. V. Subbotin and R. S. Smith. Design of distributed decentralized estimators for formations with fixed and stochastic communication topologies. Automatica, 45(11):2491 - 2501, 2009.

[10] V. Ugrinovskii. Distributed robust filtering with $H_{\infty}$ consensus of estimates. Automatica, 47(1):1 - 13, 2011.

[11] V. Ugrinovskii. Conditions for detectability in distributed consensusbased observer networks. In Proc. 51st IEEE CDC, Maui, Hawaii, 2012.

[12] V. Ugrinovskii and C. Langbort. Distributed $H_{\infty}$ consensus-based estimation of uncertain systems via dissipativity theory. IET Control Theory \& App., 5(12):1458-1469, 2011.

[13] W. M. Wonham. Linear Multivariable Control: A Geometric Approach. Springer-Verlag, New York, 3rd edition, 1985. 